\title{
Determination of the Relaxation Time of Physico-Chemical Characteristics of Water After Cavitation Treatment
}

\author{
Veronika V. Shelenkova, Tatiana A. Kulagina, \\ Olesya P. Stebeleva and Ekaterina S. Sapozhnikova \\ Siberian Federal University \\ Krasnoyarsk, Russian Federation
}

Received 25.05.2021, received in revised form 03.07.2021, accepted 01.08.2021

\begin{abstract}
The results of hydrodynamic treatment of water in the supercavitation mode are presented. Changes in the physicochemical properties of water (electrical conductivity, $\mathrm{pH}$, oxidation-reduction potential (ORP), oxygen content (OCP), etc.) were revealed, the rational duration of cavitation treatment of water was established, and data on the relaxation of these properties were obtained. At the same time, there is an increase in temperature, $\mathrm{pH}$ and electrical conductivity, a decrease in ORP and CRC. The time range with the most intense changes in $\mathrm{pH}$ and ORP is $30-90 \mathrm{~s}$. The rate of $\mathrm{pH}$ and ORP change in the range of 30-90 s is three and two times higher, respectively, than in the range of 90-300 s. These results correlate fairly well with the results of studies of the effect of ultrasound on water. It was found that the maximum $\mathrm{pH}$ value is reached under cavitation action for $180 \mathrm{~s}$. The minimum value of ORP takes at $300 \mathrm{~s}$ of cavitation treatment, but remains in the area of positive values. It is shown that during the relaxation time, activated water after treatment for 200 min under the used modes of hydrodynamic cavitation treatment does not return to its original state in all measured parameters.
\end{abstract}

Keywords: supercavitation, oxygen content, physicochemical properties of water, redox potential, electrical conductivity. 


\title{
Определение времени релаксации
}

\section{физико-химических характеристик воды}

после кавитационной обработки

\author{
В.В. Шеленкова, Т. А. Кулагина, \\ О. П. Стебелева, Е. С. Сапожникова \\ Сибирский федеральный университет \\ Российская Федераиия, Красноярск
}

\begin{abstract}
Аннотация. Изложены результаты гидродинамической обработки воды в режиме суперкавитации. Выявлены изменения физико-химических свойств воды (электропроводности, водородного показателя $\mathrm{pH}$, окислительно-восстановительного потенциала (ОВП), кислородосодержания (КРК) и др.), установлена рациональная продолжительность кавитационной обработки воды и получены данные о релаксации этих свойств. При этом наблюдается увеличение температуры, $\mathrm{pH}$ и электропроводности, уменьшение ОВП и КРК. Временной диапазон с наиболее интенсивным изменением $\mathrm{pH}$ и ОВП составляет 30-90 с. Скорость изменения величины рН и ОВП в интервале 30-90 с в три и два раза выше соответственно, чем в интервале 90-300 с. Эти результаты достаточно хорошо коррелируют с результатами исследования влияния ультразвука на воду. Обнаружено, что максимальное значение $\mathrm{pH}$ достигается при кавитационном воздействии в течение $180 \mathrm{c}$. Минимальное значение ОВП принимает при 300 с кавитационной обработки, но остается в области положительных значений. Показано, что за время релаксации активированная вода после обработки в течение 200 мин при используемых режимах гидродинамической кавитационной обработки по всем измеряемым параметрам не возвращается в исходное состояние.
\end{abstract}

Ключевые слова: суперкавитация, кислородосодержание, физико-химические свойства воды, окислительно-восстановительный потенциал, электропроводность.

Цитирование: Шеленкова, В. В. Определение времени релаксации физико-химических характеристик воды после кавитационной обработки / В. В. Шеленкова, Т. А. Кулагина, О. П. Стебелева, Е. С. Сапожникова // Журн. Сиб. федер. ун-та. Техника и технологии, 2021, 14(5). С. 550-563. DOI: 10.17516/1999-494X-0332

\section{Введение}

При эксплуатации объектов использования атомной энергии, при добыче и переработке природных ископаемых (нефти, газа, цветных металлов и т. п.) происходит поступление в окружающую среду радиоактивных изотопов в виде аэрозолей, паров, газов. В связи с этим наблюдаются значительные уровни загрязнения ими поверхностей помещений, оборудования и средств индивидуальной защиты. Уровни загрязнения радиоактивными веществами поверхностей значительно возрастают при ремонтных и аварийных работах. В этих условиях загрязненные поверхности представляют собой потенциальный источник внешнего и внутреннего облучения персонала [1, 2].

Высокие тарифы на захоронение радиоактивных отходов делают экономически неразрешимой задачу прямого захоронения всех РАО без предварительной их дезактивации, снижения категории активности или уменьшения их количества. В то же время представляет существенный интерес повторное использование высококачественного металла после дезактивации до допустимых значений активности. Данных по обращению с радиоактивными отходами и загрязнением оборудования, соответствующих помещений и сооружений на современном этапе недостаточно

$$
-551-
$$


для обеспечения требований безопасности для внешней среды на протяжении всего времени существования радионуклидов.

Преодоление сложившейся ситуации возможно с использованием дезактивирующих растворов на основе воды, обработанной с помощью эффектов кавитации, - кавитационной технологии [3-5]. Отсюда целью данной работы стало исследование физико-химических свойств воды (температуры, окислительно-восстановительного потенциала, водородного показателя, электропроводности, концентрации растворенного кислорода) после гидродинамической обработки в условиях развитой кавитации на основе феноменологической модели гидродинамического кавитационного воздействия на водные системы [6].

Следует отметить, что гидродинамические задачи для парогазового кавитационного пузырька в окружающей его жидкости играют определяющую роль в понимании механизма кавитации [7, 8], гидроакустики [9], теплофизики [8-12] и в других разделах современной науки. Они связаны также с широкими техническими приложениями, как то: работа пузырьковых камер, используемых в ядерных исследованиях, по физике высоких энергий; газожидкостные гидрореактивные двигатели и стартовые пуски ракет из-под воды; подводные взрывы (в том числе и атомные); барботажные системы различных технологических процессов; кавитационный шум и эрозия корабельных движителей и быстроходных гидромашин и аппаратов; кавитационные реакторы и эмульгаторы; аэрация скоростных потоков высоконапорных гидротехнических сооружений; аэрация акватории рек, воды в естественных и искусственных водоемах и многое другое. Известен целый ряд работ, посвященных изучению физико-химических свойств воды в различных аспектах в зависимости от потребностей промышленных производств [13-27], в том числе и в атомной отрасли $[28,29]$.

\section{Методы}

Объектом исследования данной работы являлась водопроводная и дистиллированная вода. Дистиллированная вода соответствовала ГОСТ 6709-72. Для оценки эффективности кавитационной обработки использовался ряд физико-химических показателей воды: температура, концентрация растворенного кислорода (КРК), показатель рН, электропроводность, окислительно-восстановительный потенциал. Значение водородного показателя $\mathrm{pH}$ варьировалось в пределах 5,0-6,6, удельная электропроводность не превышала 5,5 мкОм-1/см. Температура окружающей среды оставалась постоянной на протяжении всего времени эксперимента и составляла $(23 \pm 2){ }^{\circ} \mathrm{C}$. Образцы воды с момента получения дистиллята находились в стеклянной посуде, сверху неплотно закрытой тонкой полиэтиленовой пленкой, в течение не менее 3 сут. Обработка дистиллята производилась с помощью кавитационного миксера в режиме суперкавитации $(\chi=0,05)$ при частоте вращения ротора $n=10000$. Временной интервал кавитационной обработки составлял от 30 до 300 с с шагом 30 с по методике, описанной в работе [10].

На рис. 1 показан внешний вид кавитационного миксера с блоком управления и питания. Мощность двигателя составляет 1 кВт, объем рабочей камеры $3 \cdot 10^{-4} \mathrm{M}^{3}$. В качестве рабочего органа используется двухлопастная крыльчатка с клиновидным профилем, с углами раскрытия клина от 10 до $90^{\circ}$. Рабочие числа оборотов регулируются до 14000 об/мин, что обеспечивает получение чисел кавитации до $\chi=0,05$. Схема управления позволяет плавно изменять частоту

$$
-552-
$$




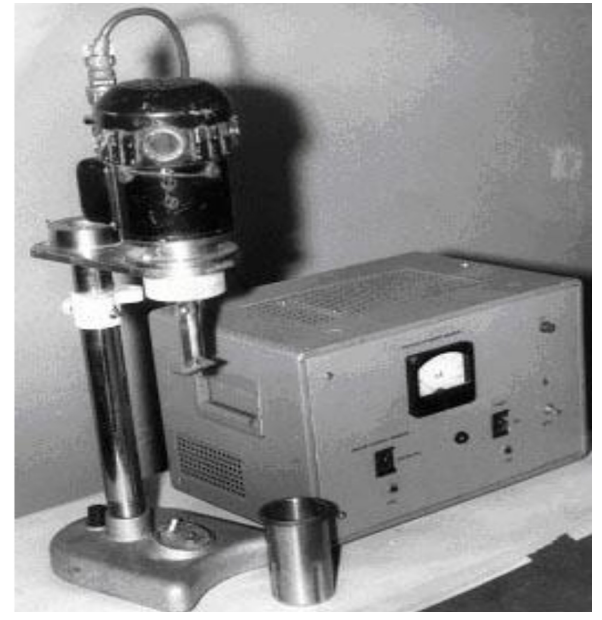

Рис. 1. Общий вид суперкавитационного миксера

Fig.1. General view of the supercavitation mixer

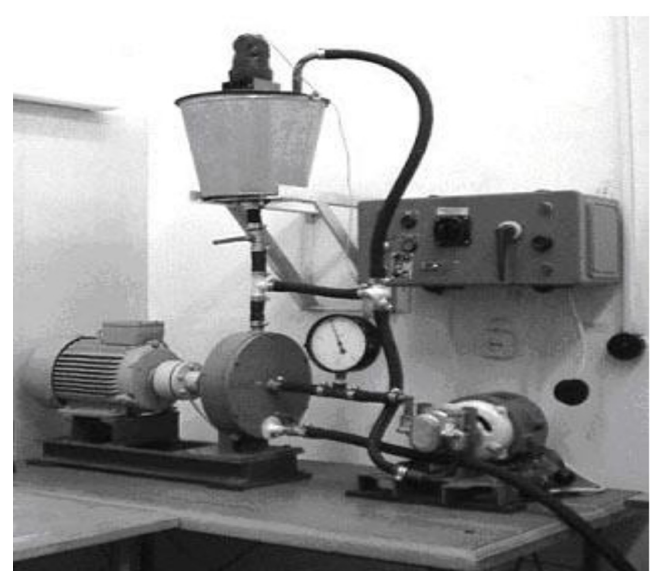

Рис. 2. Внешний вид экспериментального стенда

Fig.2. External view of the experimental stand

вращения ротора, поддерживать число оборотов независимо от изменяющейся нагрузки и фиксировать время обработки.

На рис. 2 и 3 показаны внешний вид и схема стенда. Мощность электродвигателя 4 кВт, объем рабочей камеры составляет $1,5 \cdot 10^{-2} \mathrm{M}^{3}$, скорость вращения четырехлопастного клиновидного кавитатора составляет 4000 об/мин. Для измерения физико-химических показателей воды использовался анализатор растворенного кислорода «МАРК-201» и 4-параметровый прибор «WaterTest». Оценка погрешности подтвердила удовлетворительную точность полученных результатов.

Время между кавитационным воздействием на воду и измерением значения исследуемого параметра активированной воды составляло не более двух минут. Временной интервал для изучения релаксации активированной воды в исходное состояние составлял от 200 до 300 мин с шагом в 5 мин для образцов со временем обработки 30, 60, 90 и 300 с. Средние значения рН, электропроводности $(S)$, ОВП (окислительно-восстановительный потенциал), температуры $T$ вычислялись по пяти измерениям каждой экспериментальной точки. Эксперименты с водопроводной водой, отстоявшейся (1 и 5 сут) и неотстоявшейся (10-15 мин), проводились аналогично экспериментам с дистиллированной водой.

Для определения графических и функциональных зависимостей физико-химических характеристик воды от различных параметров был использован метод регрессионного анализа.

\section{Результаты и обсуждения}

На основании результатов работы [13] был выбран режим суперкавитации $(\chi=0,05)$ при постоянной частоте вращения ротора $n=10000$ об/мин. Обрабатываемый объем воды был равен 100 мл. На рис. 4 и 5 представлены результаты измерений в виде графических зависимостей физико-химических свойств дистиллированной воды от времени кавитационной обработки. Видно, что с увеличением времени обработки наблюдается повышение температуры воды более чем на $40 \%$. 


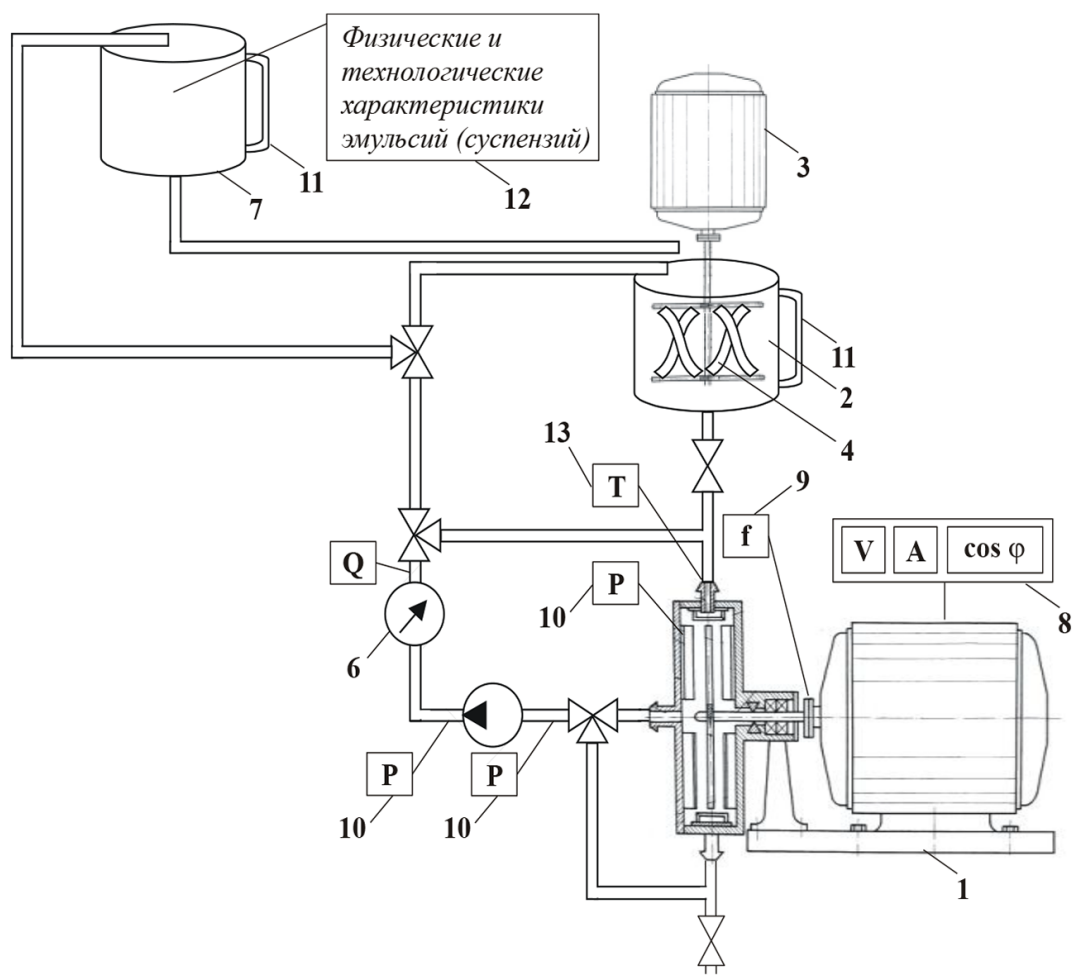

Рис. 3. Схема стенда для кавитационной обработки воды: 1 - кавитационный смеситель; 2 - емкость с исходным продуктом; 3 - электродвигатель; 4 - механический гомогенизатор; 5 - насос; 6 - расходомер; 7 -контрольная емкость; 8 - блок измерения электрических параметров привода кавитационного аппарата; 9 - блок измерения частоты вращения; 10 - блок измерения давления; 11 - уровнемер; 12 - измерительнолабораторный комплекс для определения характеристик воды; 13 - блок измерения температуры

Fig. 3. Scheme of the stand for cavitation treatment of water: 1 - cavitation mixer; 2 - container with the original product; 3 - electric motor; 4 - mechanical homogenizer;5 - pump; 6 - flow meter; 7 - control capacity; 8 - unit for measuring electrical parameters of the drive of the cavitation apparatus; 9 - unit for measuring rotation frequency;;10 - pressure measurement unit; 11 - level gauge; 12 - measuring and laboratory complex for determining the characteristics of water; 13 - temperature measuring unit

Это связано с пульсацией и последующим коллапсом кавитационных пузырьков. Зависимость температуры от времени обработки линейная, интенсивность изменения температуры характеризуется тангенсом угла наклона прямой $\operatorname{tg} \alpha=0,04$ (рис. $4 a$ ). Полученные экспериментальные данные также хорошо согласуются с результатами работы [13]. Экспериментальная зависимость изменения КРК воды от времени кавитационного воздействия представлена на рис. 4б. Каждая точка построена по усредненному значению пяти измерений. Относительная ошибка определения КРК 2,5 \%. Концентрация кислорода в воде линейно уменьшается при увеличении времени воздействия кавитации. Интенсивность изменения КРК характеризуется тангенсом угла наклона прямой $\operatorname{tg} \alpha=0,01$.

Измерения значений КРК активированной воды в течение 200 мин с шагом в 5 мин на образцах со временем обработки 30, 60, 90 и 300 с показали, что повышение значений КРК до значений, близких к значениям дистиллированной воды, наблюдалось в течение первых 100 мин, но и при 200 мин не достигло исходного значения КРК (рис. 4 в). 


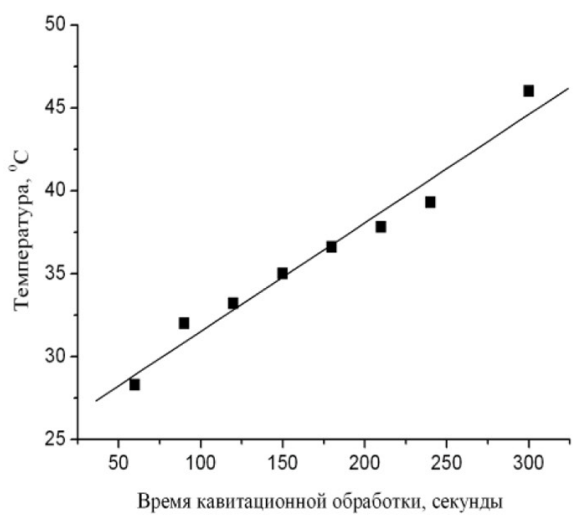

a) $y=24,99+0,04 x$

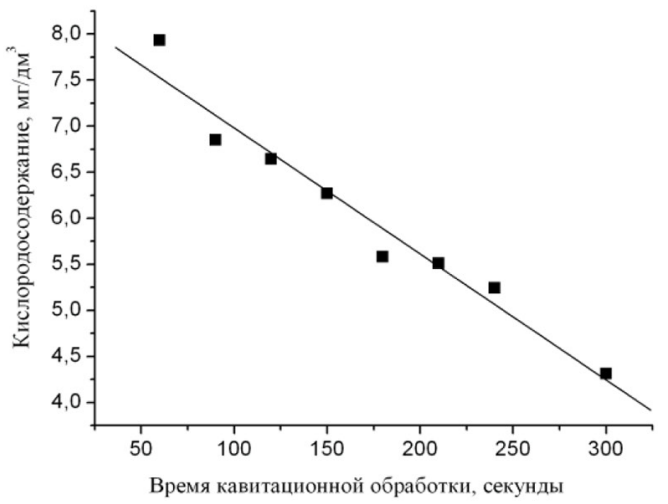

б) $y=8,35-0,01 x$

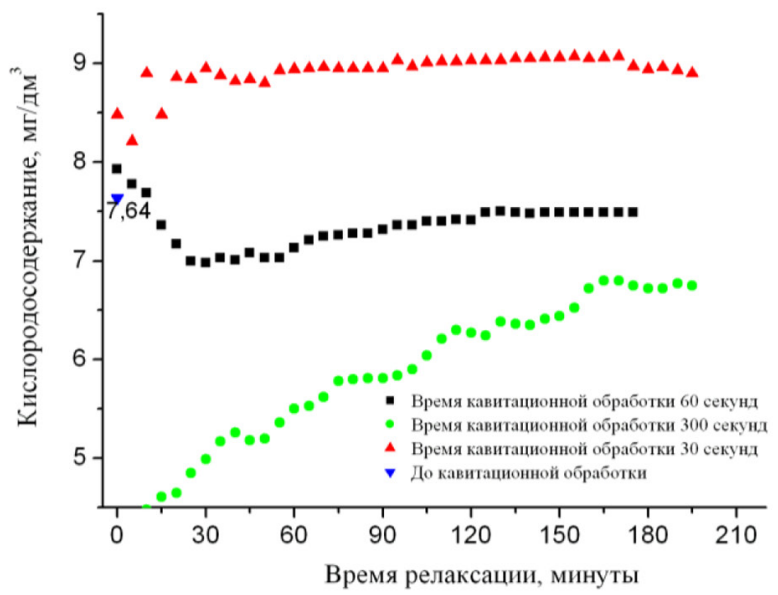

в)

Рис. 4. Изменение КРК дистиллированной воды в зависимости от времени кавитационной обработки и времени релаксации

Fig.4. Change in the CRC of distilled water depending on the time of cavitation treatment and relaxation time

На рис. 5 а представлена зависимость $\mathrm{pH}$ от времени обработки. Водородный показатель pH возрастает с увеличением времени кавитационного воздействия. При времени обработки 180 с разница между максимальным значением $\mathrm{pH}$ активированной воды и $\mathrm{pH}$ исходного дистиллята $\Delta \mathrm{pH}_{\max }$ составила 1,1 . Наиболее интенсивное изменение $\mathrm{pH}$ соответствует интервалу 30-90 с. В этом диапазоне рН повышается на 15 \%, тогда как максимальное повышение $\mathrm{pH}$ составляет 18 \%. Скорость изменения $\mathrm{pH}$ при кавитационном воздействии в диапазоне 30 90 с почти в два раза превышает скорость изменения рН при воздействии в диапазоне 90-300 с. После кавитационного воздействия проводились измерения значений $\mathrm{pH}$ активированной воды в течение 200 мин с шагом в 5 мин на образцах со временем обработки 30, 60, 90 и 300 с. В течение этого времени показатели рН для всех образцов не вернулись к первоначальному значению.

Динамика поведения ОВП в процессе кавитационной обработки воды представлена на рис. 5 б. Относительная ошибка определения ОВП $2 \%$. На графике наблюдается участок наиболее интенсивного изменения ОВП в интервале 30-90 с. ОВП понижается на 43 \%, тог- 

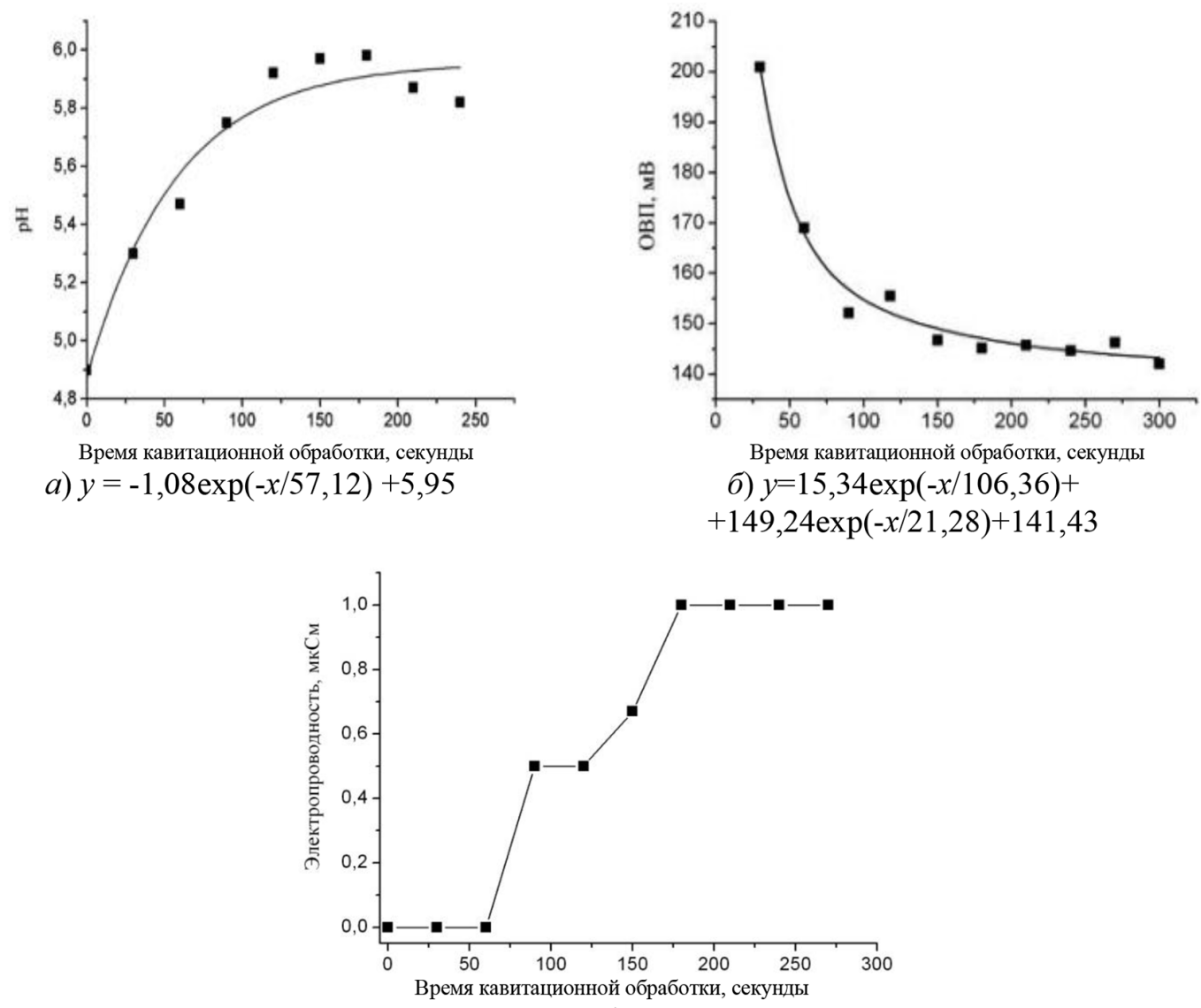

b)

Рис. 5. Изменение физико-химических параметров дистиллированной воды в зависимости от времени кавитационной обработки

Fig. 5. Change of physicochemical parameters of distilled water depending on the time of cavitation treatment

да как максимальное понижение ОВП составляет $47 \%$, но не принимает отрицательных значений. Скорость изменения ОВП при кавитационном воздействии в диапазоне 30-90 с почти в три раза превышает скорость изменения ОВП при воздействии в диапазоне 90-300 с. Следует отметить, что в работе [30] при воздействии ультразвуком на воду обнаружено, что оптимальное изменение $\mathrm{pH}$ воды наблюдается при обработке в течение одной минуты. Проводились измерения значений ОВП активированной воды в течение $~ 200$ мин с шагом в 5 мин на образцах со временем обработки 30, 60, 90 и 300 с. Повышение ОВП до значений, близких к значениям дистиллированной воды, наблюдалось в первые 40 мин.

В серии экспериментов по выявлению воздействия на дистиллированную воду кавитационных эффектов различной продолжительности (30-300 с) было установлено повышение электропроводности (рис. 5 в). Значение электропроводности исходного дистиллята составляло 0 мкОм $^{-1} / \mathrm{cm}$, что связано с низкой чувствительностью прибора. Электропроводность активированной воды в течение 200 мин с шагом в 5 мин на образцах со временем обработки 30 , 60 и 90 с не изменялась и оставалась равной 1 мкОм-1/см.

$$
-556-
$$


Исследования физико-химических параметров водопроводной воды проводились аналогично исследованиям для дистиллированной воды: число кавитации $\chi=0,05$; частота вращения ротора $n=10000$ об/мин; обрабатываемый объем воды составлял 100 мл. В эксперименте была использована водопроводная вода с различным временем отстаивания (время после ее набора): 0,1 и 5 сут. Результаты экспериментов представлены графически на рис. 6, а уравнения регрессии приведены в табл. 1.

При кавитации с увеличением времени обработки наблюдается повышение температуры воды примерно на 30 \% для трех образцов (рис. $6 a$ ). Зависимости температур от времени обработки линейные, по мере увеличения времени отстаивания воды интенсивность изменения температур уменьшается: $\operatorname{tg} \alpha=0,02-0,04$. Из графических зависимостей можно выделить общую точку для трех прямых - при кавитационном воздействии на воду в течение 180 с.

Значение электропроводности воды при кавитации увеличивалось при увеличении времени действия кавитации и времени отстаивания. Наиболее интенсивное изменение электропроводности наблюдалось для неотстоявшейся воды (рис. 6б). Динамика поведения ОВП в процессе кавитационной обработки воды представлена на рис. 6в. Относительная ошибка

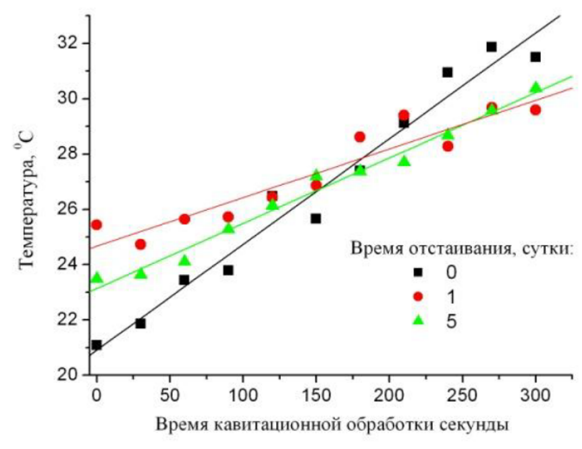

a)

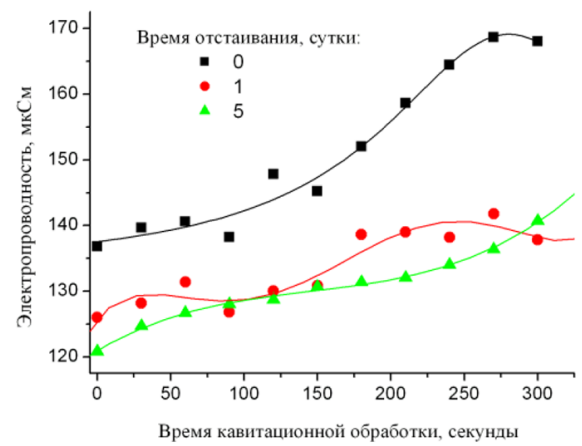

б)

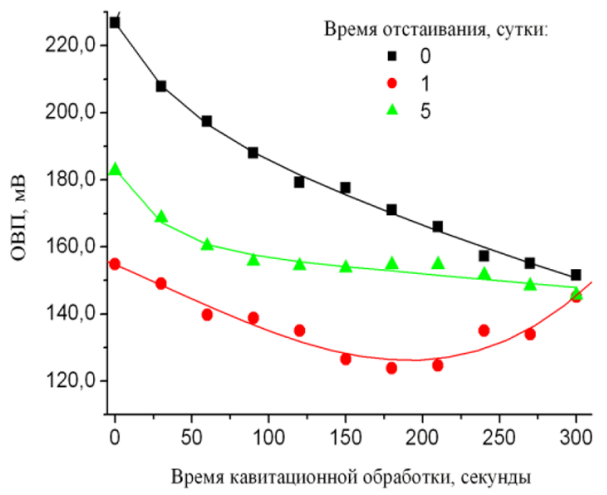

в)

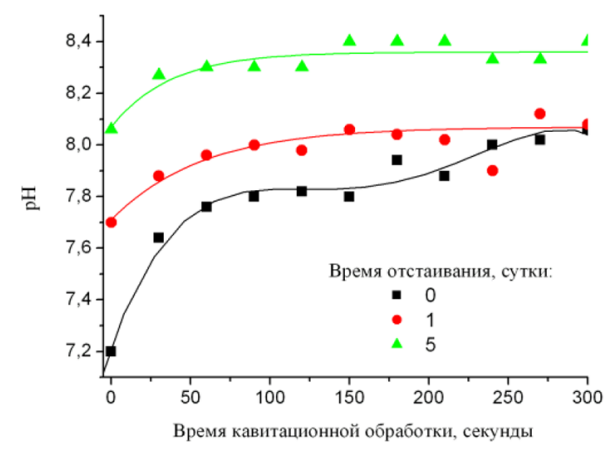

2)

Рис. 6. Зависимости физико-химических параметров водопроводной воды, не отстоявшейся и отстоявшейся в течение 1 и 5 сут, от времени кавитационной обработки

Fig. 6. Dependences of the physicochemical parameters of tap water not settled and settled for 1 and 5 days on the time of cavitation treatment 
Таблица 1. Уравнения полиномов, аппроксимирующие зависимости физико-химических параметров активированной водопроводной воды, не отстоявшейся и отстоявшейся в течение 1 и 5 сут

Table 1. Equations of polynomials approximating the dependences of the physicochemical parameters of activated tap water not settled and settled for 1 and 5 days

\begin{tabular}{|l|l|l|}
\hline $\begin{array}{c}\text { Уравнения } \\
\text { регрессии }\end{array}$ & \multicolumn{1}{|c|}{$V=100$ мл.; $\chi=0,05$} & \multicolumn{1}{c|}{$V=1000$ мл.; $\chi=0,01$} \\
\hline$T$ & $y=25,37-0,02 x+2,13 x^{2}+1,77 x^{3}-2,23 x^{4}$ & $y=23,69+0,22 x-8,25 x^{2}$ \\
\hline OВП & $y=154,66-0,19 x-2,98 x^{2}+2,81 x^{3}$ & $y=144,44-2,85 x+0,05 x^{2}-2,07 x^{3}$ \\
\hline$S$ & $y=125,70+0,23 x-0,004 \mathrm{x}^{2}+3,67 x^{3}-1,16 x^{4}+1,24 x^{5}$ & $y=122,56+0,67 x-0,002 x^{2}$ \\
\hline $\mathrm{pH}$ & $y=-0,36 \exp (-x / 56,90)+8,07$ & $y=-0,58 \exp (-x / 40,81)+7,95$ \\
\hline
\end{tabular}

определения ОВП 3-5 \%. На графике наблюдается участок наиболее интенсивного изменения ОВП в интервале 30-220 с для неотстоявшейся воды и 30-90 с для отстоявшейся в течение 1 и 5 сут. ОВП понижается на 23,9 и 20 \% соответственно, но не принимает отрицательных значений. Скорость изменения ОВП при кавитационном воздействии в диапазоне 30-90 с почти в три раза превышает скорость изменения ОВП при воздействии в диапазоне 90-300 с для отстоявшейся воды.

На рис. 62 представлена зависимость $\mathrm{pH}$ от времени обработки. Водородный показатель pH повышается с увеличением времени кавитационного воздействия. Наиболее интенсивное изменение $\mathrm{pH}$, как и в случае с дистиллированной водой, соответствует интервалу 30-90 с. В этом диапазоне $\mathrm{pH}$ повышается на 4 \%, что совпадает с его максимальным повышением. В диапазоне 90-300 с значение рН принимает постоянное значение.

Кроме того, для водопроводной воды проведен ряд исследований параметров при другом режиме кавитации: число кавитации $\chi=0,01$; частота вращения ротора $n=2880$ об/мин; обрабатываемый объем воды составлял 1000 мл. На рис. 7 представлены результаты измерений физико-химических свойств воды, отстоявшейся в течение 1 сут. Также для сравнения действия кавитации при двух режимах кавитации приведены зависимости параметров воды, описанных выше. По уравнениям регрессии (табл. 2) видно, что полиномы для параметров ОВП и $\mathrm{pH}$ имеют подобную структуру и отличаются лишь коэффициентами. Более интенсивное изменение всех параметров наблюдалось для режима кавитации с $\chi=0,01$.

Таблица 2. Уравнения полиномов, аппроксимирующих зависимости физико-химических параметров активированной водопроводной воды, отстоявшейся в течение 1 сут

Table 2. Equations of polynomials approximating the dependences of the physicochemical parameters of activated tap water distilled for 1 day

\begin{tabular}{|l|l|l|}
\hline \multicolumn{1}{|c|}{$\begin{array}{c}\text { Уравнения } \\
\text { регрессии }\end{array}$} & \multicolumn{1}{|c|}{$V=100$ мл. $\chi=0,05$} & \multicolumn{1}{c|}{$V=1000$ мл. $\chi=0,01$} \\
\hline$T$ & $y=25,37-0,02 x+2,13 x^{2}+1,77 x^{3}-2,23 x^{4}$ & $y=23,69+0,22 x-8,25 x^{2}$ \\
\hline OВП & $y=154,66-0,19 x-2,98 x^{2}+2,81 x^{3}$ & $y=144,44-2,85 x+0,05 x^{2}-2,07 x^{3}$ \\
\hline$S$ & $y=125,70+0,23 x-0,004 x^{2}+3,67 x^{3}-1,16 x^{4}+1,24 x^{5}$ & $y=122,56+0,67 x-0,002 x^{2}$ \\
\hline $\mathrm{pH}$ & $y=-0,36 \exp (-x / 56,90)+8,07$ & $y=-0,58 \exp (-x / 40,81)+7,95$ \\
\hline
\end{tabular}




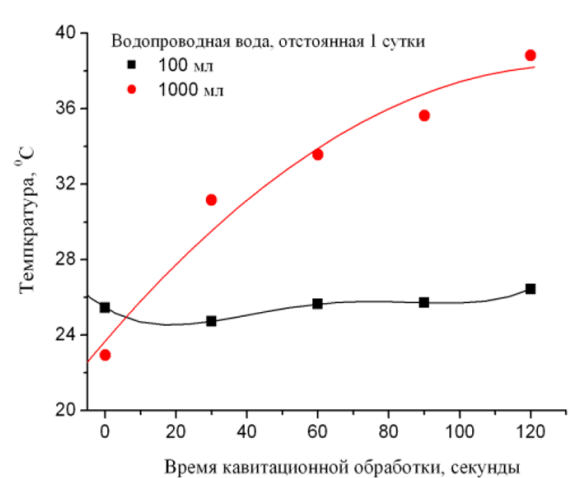

a)

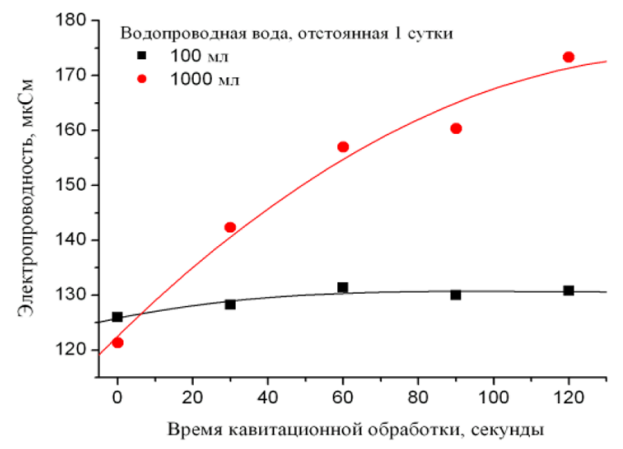

б)

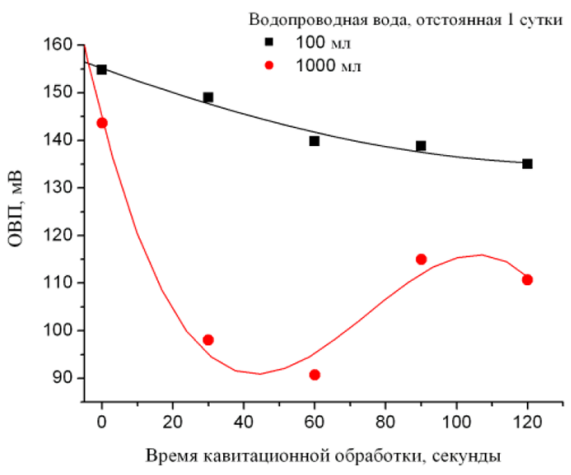

b)

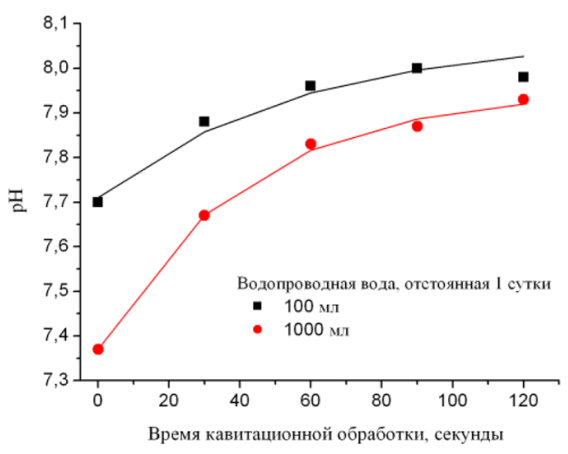

2)

Рис. 7. Зависимости физико-химических параметров водопроводной воды, отстоявшейся в течение 1 сут, от времени кавитационной обработки и обрабатываемого объема

Fig. 7. Dependences of the physicochemical parameters of tap water, settled for 1 day, on the time of cavitation treatment and the processed volume

Для режима кавитации $\chi=0,01$ проводились измерения значений ОВП, $\mathrm{pH}$ и электропроводности активированной неотстоявшейся воды в течение 200 мин с шагом в 5 мин и в течение 4-х сут с шагом 1 сут на образцах со временем обработки 30, 60 и 150 с. Наблюдалось интенсивное понижение ОВП в течение двух суток, после чего величина ОВП приняла постоянное значение. Значение электропроводности и рН в течение 1-2 сут постоянное, но даже и по истечении пяти суток не возвращается к исходной величине до воздействия кавитации.

\section{Заключение}

Проведено экспериментальное изучение реакции воды на гидродинамическое кавитационное воздействие при числах кавитации $\chi=0,05 ; 0,01$ и числах оборотов ротора $\mathrm{n}=10000$ и 2880 об/мин в зависимости от времени воздействия. Выявлены изменения физикохимических свойств воды (электропроводности, водородного показателя $\mathrm{pH}$, окислительновосстановительного потенциала, кислородосодержания и др.), установлена рациональная продолжительность кавитационной обработки воды. При этом наблюдается увеличение Т, pH и электропроводности, уменьшение ОВП и КРК. 
Временной диапазон с наиболее интенсивным изменением рН и ОВП составляет 30-90 с. Скорость изменения величины $\mathrm{pH}$ и ОВП в интервале 30-90 с в три и два раза выше соответственно, чем в интервале 90-300 с. Эти результаты достаточно хорошо коррелируют с результатами исследования влияния ультразвука (44 кГц) на воду.

Обнаружено, что максимальное значение $\mathrm{pH}$ достигается при кавитационном воздействии в течение 180 с. Минимальное значение ОВП принимает при 300 с кавитационной обработки, но остается в области положительных значений.

Показано, что за время релаксации активированная вода после обработки в течение 200 мин при используемых режимах гидродинамической кавитационной обработки по всем измеряемым параметрам (Т, КРК, $\mathrm{pH}, \mathrm{OBП}, S$ ) не возвращается в исходное состояние.

Полученные результаты могут быть использованы для управления физико-химическими свойствами воды с помощью изменения режимов кавитации. А это, в свою очередь, может способствовать более эффективному использованию активированной воды в различных отраслях промышленности.

\section{Благодарность / Acknowledgement}

Исследование выполнено при финансовой поддержке РФФИ, Правительства Красноярского края и Красноярского краевого фонда науки в рамках научного проекта № 20-48-243001.

The research was funded by RFBR, Krasnoyarsk Territory and Krasnoyarsk Regional Fund of Science, project number 20-48-243001.

\section{Список литературы/ References}

[1] Шеленкова В.В., Кормич А.И., Козин О.А., Кулагина Т.А. Дезактивация оборудования от радиоактивного загрязнения водой после кавитационной обработки. Журнал СФУ. Техника и технологии. 2018. 11(6). 732-743; DOI: 10.17516/1999-494X-0088 [Shelenkova V.V., Kormich A.I., Kozin O.A., Kulagina T.A. Decontamination of equipment from radioactive contamination with water after cavitation treatment. J. Sib. Fed. Univ. Eng. Technol. 2018.11(6). 732-743 (in Russian)].

[2] Гафарова В. В. (Шеленкова В. В.), Кулагина Т. А. Безопасные методы утилизации радиоактивных отходов. Журнал СФУ. Техника и технологии. 2016. 9(4). 585-597; DOI: 10.17516/1999494X-2016-9-4-585-597 [V. V. Gafarova (Shelenkova V. V.), Kulagina T. A. Safe methods of disposal of radioactive waste. J. Sib. Fed. Univ. Eng. Technol. 2016. 9(4). 585-597 (in Russian)].

[3] Шеленкова В.В., Кулагина Т.А. Совершенствование технологии дезактивации оборудования с радиоактивным загрязнением. Радиоактивные отходы. 2021. № 1(14). 28-38. DOI: 10.25283/2587-9707-2021-1-28-38 [Shelenkova V.V., Kulagina T. A. Improvement of technology for decontamination of equipment with radioactive contamination. Radioactive waste. 2021. 1(14). 28-38. (in Russian)].

[4] Шеленкова В.В., Кулагина Т.А. Изучение возможности дезактивации загрязненных образцов растворами на основе кавитационно-активированной воды. XXI век. Техносферная безопасность. 2018. 3(3). 36-43 [Shelenkova V.V., Kulagina T. A. Study of the possibility of decontamination of contaminated samples with solutions based on cavitation-activated water. XXI Century. Technosphere safety. 2018. 3(3). 36-43 (in Russian)]. 
[5] Кулагина Т. А., Шеленкова В. В. Способы дезактивации поверхностей с радиоактивным загрязнением. Журнал СФУ. Техника и технологии. 2017. 10(3). 352-363; DOI: 10.17516/1999494X-2017-10-3-352-363 [Kulagina T. A. Shelenkova V.V. Methods for decontamination of surfaces with radioactive contamination. J. Sib. Fed. Univ. Eng. Technol. 2017. 10(3). 352-363 (in Russian)].

[6] Кулагин В. А., Кулагина Т. А., Шеленкова В. В. Феноменологическая модель гидродинамического кавитационного воздействия на водные системы. Журнал СФУ. Техника и технологии. 2019. 12(7). 818-829. DOI: 10.17516/1999-494X-0182 [Kulagin V. A., Kulagina T. A., Shelenkova V. V. Phenomenological model of hydrodynamic cavitation impact on water systems. J. Sib. Fed. Univ. Eng. Technol. 2019. 12(7). 818-829 (in Russian)].

[7] Ивченко В.М., Кулагин В.А., Немчин А.Ф. Кавитационная технология; ред. акад. Г.В. Логвинович. Красноярск: Изд-во КГУ, 1990. 200 с. [Ivchenko V.M., Kulagin V.A., Nemchin A. F. Cavitation technology; ed. acad. G. V. Logvinovich. Krasnoyarsk: Publishing house of KSU, 1990. 200 p. (in Russian)].

[8] Демиденко Н.Д., Кулагин В. А., Шокин Ю. И., Ли Ф.-Ч. Тепломассообмен и суперкавитаиия. Новосибирск: Наука, 2015. 436 с. ISBN978-5-02-019206-5 [Demidenko N.D., Kulagin V.A., Shokin Yu.I., Li F.-Ch. Heat and mass transfer and supercavitation. Novosibirsk: Nauka, 2015. 436 p. (in Russian)].

[9] Kulagin V.A., Kulagina L.V., Li Feng-Chen. Fundamentals of Cavitation Treatment for Multicomponent Environments. M.: RU-SCIENCE, 2017. 182 p. ISBN978-5-4365-1862-6.

[10] Кулагин В.А. Методы и средства технологической обработки многокомпонентных сред с использованием эффектов кавитации: Дисс. ... д-ра. техн. наук. Красноярск, 2004. 379 c. [Kulagin V.A. Methods and means of technological processing of multicomponent media using cavitation effects: Diss. ... doct. tech. sciences. Krasnoyarsk, 2004. 379 p. (in Russian)].

[11] Балабышко А.М., Зимин А.И., Ружицкий В.П. Гидромеханическое диспергирование. M.: Наука, 1998. 330 c.[Balabyshko A. M., Zimin A. I., Ruzhitskiy V.P. Hydromechanical dispersion. Moscow: Nauka, 1998. 330 p. (in Russian)].

[12] Кнепп Р., Дейли Д., Хеммит Ф. Кавитаиия. М.: Мир, 1974. 678 с. [Knepp R., Daley D., Hemmit F. Cavitation. Moscow: Mir, 1974. 678 p. (in Russian)].

[13] Криволуцкий А.С., Кулагин В.А. Изменение физико-химических свойств воды под воздействием гидродинамической кавитации. Сочиальные проблемы инженерной экологии, природопользования и ресурсосбережения: Матер. НПК. Красноярск: ИПЦ КГТУ, 2003. 61-74 [Krivolutskiy A. S., Kulagin V. A. Changes in the physicochemical properties of water under the influence of hydrodynamic cavitation. Social problems of engineering ecology, nature management and resource conservation: Mater. NPK. Krasnoyarsk: IPC KSTU, 2003. 61-74 (in Russian)].

[14] Криволуцкий А.С., Кулагин В.А. Релаксация физико-химических свойств воды, прошедшей обработку гидродинамической кавитацией. Социальные проблемы инженерной экологии, природопользования и ресурсосбережения: Матер. НПК. Красноярск: ИПЦ КГТУ, 2003. 22-29 [Krivolutskiy A.S., Kulagin V.A. Relaxation of the physicochemical properties of water treated by hydrodynamic cavitation. Social problems of engineering ecology, nature management and resource conservation: Mater. NPK. Krasnoyarsk: IPCKSTU, 2003. 22-29 (inRussian)]. 
[15] Криволуцкий А. С., Кулагин В.А. Влияние масштабных факторов при различных режимах кавитационной обработки воды. Вестник ассоциации выпускников КГТУ, Красноярск. 2010. Вып. 19. 70-75 [Krivolutskiy A. S., Kulagin V. A. Influence of large-scale factors in different modes of cavitation treatment of water. Bulletin of the KSTU Alumni Association, Krasnoyarsk. 2010. Issue. 19. 70-75 (in Russian)].

[16] Сапожникова Е.С., Кулагин В.А., Кашкина Л.В., Стебелева О.П. Влияние гидродинамической кавитационной обработки на физико-химические свойства воды. ВНКСФ-17: Матер. Всерос. научной конференции студентов-физиков. Екатеринбург, 2011. 239-241 [Sapozhnikova E.S., Kulagin V.A., Kashkina L.V., Stebeleva O.P. Influence of hydrodynamic cavitation treatment on the physicochemical properties of water. VNKSF-17: Mater. Vseros. scientific conference of physics students. Yekaterinburg, 2011. 239-241 (in Russian)].

[17] Стебелева О.П., Сапожникова Е.С., Криволуцкий А.С. Исследование физикохимических характеристик воды при различных временных режимах кавитации. Вестник ассоциации выпускников КГТУ. Красноярск, 2011. 78-83 [Stebeleva O.P., Sapozhnikova E. S., Krivo lutskiy A.S. Investigation of the physicochemical characteristics of water at various time regimes of cavitation. Bulletin of the KSTU Alumni Association. Krasnoyarsk, 2011. 78-83 (in Russian)].

[18] Сапожникова Е.С., Кулагин В.А., Кашкина Л.В., Стебелева О.П. Исследование кавитационной воды. Тезисы XVI Всерос. симп. с междунар. участием «Сложные системы в экстремальных условиях». Красноярск, 22-25 мая 2012. 83[Sapozhnikova E. S., Kulagin V.A., Kashkina L.V., Stebeleva O.P. Studyofcavitationwater. Abstracts of the XVI All-Russia. Simp. with int. participation «Complex systems in extreme conditions». Krasnoyarsk, May 22-25, 2012. 83. (in Russian)].

[19] Сапожникова Е.С., Кулагин В.А., Кашкина Л. В., Стебелева О. П. Динамика изменения свойств воды в процессе кавитационной гидродинамической обработки. 21 век: фундаментальная наука и технологии: материалы международной научно-практической конференциии. М., 2012. 121-126 [Sapozhnikova E. S., Kulagin V. A., Kashkina L. V., Stebeleva O. P. Dynamics of changes in water properties during cavitation hydrodynamic treatment. 21st century: fundamental science and technology: materials of the international scientific and practical conference. M., 2012. 121-126 (inRussian)].

[20] Кулагин В. А., Сапожникова Е. С., Стебелева О.П. и др. Особенности влияния эффекта кавитации на физико-химические свойства воды и стоков. Журнал СФУ. Техника и технологии. 2014. 7(5). 605-614 [Kulagin V. A., Sapozhnikova E. S., Stebeleva O. P. and other Features of the influence of the effect of cavitation on the physicochemical properties of water and wastewater. J. Sib. Fed. Univ. Eng. Technol. 2014. 7(5). 605-614 (in Russian)].

[21] Дубровская О. Г., Сапожникова Е. С., Кулагин В. А. Современные компоновки технологических схем очистки сточных вод с использованием кавитационной технологии. Журнал СФУ. Техника и технологии. 2015. 8(2). 217-223 [Dubrovskaya O.G., Sapozhnikova E. S., Kulagin V. A. Modern layouts of technological schemes for wastewater treatment using cavitation technology J. Sib. Fed. Univ. Eng. Technol. 2015. 8 (2). 217-223 (in Russian)].

[22] Дубровская О.Г., Сапожникова Е.С., Кулагин В.А. и др. Математическое моделирование кавитационных процессов при кондиционировании промышленных сточных вод. Журнал СФУ. Техника и технологии. 2015. 8(3). 369-376 [Dubrovskaya O.G., Sapozhnikova E. S., 
Kulagin V. A. et al. Mathematical modeling of cavitation processes in the conditioning of industrial waste water. J. Sib. Fed. Univ. Eng. Technol. 2015. 8(3). 369-376 (in Russian)].

[23] Евстигнеев В. В., Кулагин В. А. Кавитация в технологиях очистки сточных вод. В мире научных открытий. 2010. 5(11). Ч. I. 87-90 [Evstigneev V. V., Kulagin V. A. Cavitation in wastewater treatment technologies. In the world of scientific discoveries. 2010. 5(11). Part I. $87-90$ (in Russian)].

[24] Криволуцкий А.С. Повышение эффективности работы тепловых сетей за счет кавитационной обработки воды. Дисс. ... канд. техн. наук, Красноярск: СФУ, 2007. 206 с. [Krivolutskiy A. S. Improving the efficiency of heating networks due to cavitation treatment of water. Diss. ... Cand. Tech. Sciences, Krasnoyarsk: Siberian Federal University, 2007. 206 p. (in Russian)].

[25] Дубровская О. Г. Технология гидротермодинамической обработки природных и сточных вод с использованием эффектов кавитаиии. Дисс. ... канд. техн. наук, Красноярск: СФУ, 2007. 134 c. [Dubrovskaya O.G. Technology of hydrothermodynamic treatment of natural and waste waters using cavitation effects. Diss. ... Cand. tech. Sciences, Krasnoyarsk: Siberian Federal University, 2007. 134 p. (in Russian)].

[26] Евстигнеев В.В. Совершенствование технологии кондиционирования сточных вод энергетических систем и комплексов. Дисс. ... канд. техн. наук. Красноярск: СФУ, 2012. 123 c. [Evstigneev V. V. Improvement of waste water conditioning technology for energy systems and complexes. Diss. ... Cand. tech. Sciences, Krasnoyarsk: Siberian Federal University, 2012. 123 p. (in Russian)].

[27] Пьяных Т. А. Повышение эффективности суперкавитационных испарителей. Дисс. ... канд. техн. наук, Красноярск: СФУ, 2013. 126 с. [Р'yany Т. А. Improving the efficiency of supercavitation evaporators. Diss. ... Cand. tech. Sciences, Krasnoyarsk: Siberian Federal University, 2013. 126 p. (in Russian)].

[28] Попков В.А. Развитие технологий обращения с отработавщим ядерным топливом. Дисс. ... канд. техн. наук, Красноярск: СФУ, 2016. 149 с. [Popkov V. А. Development of technologies for handling spent nuclear fuel. Diss. ... Cand. tech. Sciences, Krasnoyarsk: Siberian Federal University, 2016. 149 p. (in Russian)].

[29] Козин О.А. Методы и средства повышения экологической безопасности обращения с отходами ядерно-энергетического ичикла. Дисс. ... канд. техн. наук. Красноярск: СФУ, 2011. 123 c. [Kozin O. A. Methods and means of improving the environmental safety of nuclear waste management. Diss. ... Cand. tech. Sciences, Krasnoyarsk: Siberian Federal University, 2011. 123 p. (in Russian)].

[30] Букатый В.И., Нестерюк П.И. Измерение физико-химических характеристик воды при различных физических воздействиях с учетом переходных процессов. Ползуновский вестник. 2010. 2. 60-65 [Bukatyy V.I., Nesteruk P. I. Measurement of physicochemical characteristics of water under various physical influences, taking into account transient processes. Polzunovsky Bulletin. 2010. 2. 60-65 (in Russian)]. 